\title{
High-resolution burn imaging in pig skin by photoacoustic microscopy
}

Hao F. Zhang, Konstantin Maslov, George Stoica, Lihong V. Wang

Hao F. Zhang, Konstantin Maslov, George Stoica, Lihong V. Wang, "Highresolution burn imaging in pig skin by photoacoustic microscopy," Proc. SPIE 6437, Photons Plus Ultrasound: Imaging and Sensing 2007: The Eighth Conference on Biomedical Thermoacoustics, Optoacoustics, and Acoustooptics, 64370A (13 February 2007); doi: 10.1117/12.698005

SPIE. Event: SPIE BiOS, 2007, San Jose, California, United States 


\title{
High-resolution burn imaging in pig skin by photoacoustic microscopy
}

\author{
Hao F. Zhang ${ }^{\mathrm{a}}$, Konstantin Maslov ${ }^{\mathrm{a}}$, George Stoica ${ }^{\mathrm{b}}$, and Lihong V. Wang ${ }^{* a}$ \\ ${ }^{a}$ Department of Biomedical Engineering, Washington University in St. Louis, One Brookings Drive, \\ St. Louis, Missouri, USA 63130; \\ ${ }^{\mathrm{b}}$ Department of Veterinary Pathobiology, Texas A\&M University, 4467 TAMU, College Station, \\ Texas, USA 77843
}

\begin{abstract}
Existing noninvasive imaging modalities fail to provide high-resolution depth-resolved imaging of skin burns in large depths. Hence, the measurement of burn depth, especially for partial-thickness burn, remains inaccurate. We used photoacoustic microscopy to measure the depth of acute thermal burns by imaging the microvasculature damage. In this work, partial-thickness burns were induced in vivo on pig skin. Limited by the flexibility of the photoacoustic scanning system, photoacoustic images of the burns were acquired after skin excision. Experimental results show that burn depth increases with longer heating duration. The maximum imaged burn depth measures $\sim 1.7 \mathrm{~mm}$ with a depth resolution of $15 \mu \mathrm{m}$.
\end{abstract}

Keywords: Burn, microvasculature, photoacoustic, microscopy

\section{INTRODUCTION}

The depth of burn injury is a critical parameter for the clinical evaluation of burns. ${ }^{1}$ Skin burns are categorized by depth into superficial, partial-thickness and full-thickness burns. With both very shallow (superficial, 0.07 0.12 $\mathrm{mm}$ ) and very deep (full-thickness, >2 mm) burns, clinical assessment of the burn depth is generally accurate. However, the most complicated cases are partial-thickness burns, which range from $0.12 \mathrm{~mm}$ to $2 \mathrm{~mm}$ and are further divided into superficial partial-thickness and deep partial-thickness burns. Owing to the destruction of the epidermis and a portion of the underlying dermis, clinical decisions about partial-thickness burns is often inaccurate. ${ }^{2,3}$ Because superficial partialthickness burns heal naturally whereas deep partial-thickness burns require immediate medical attention or skin grafting, there is a need for accurate determination of burn depth by noninvasive technologies.

Existing techniques that have been investigated to measure burn depth noninvasively include laser Doppler perfusion imaging (LDPI), ${ }^{4}$ polarization sensitive optical coherence tomography (PSOCT), ${ }^{5,6}$ indocyanine green dye fluorescence imaging, thermography, ultrasound imaging, and nuclear magnetic resonance imaging. Among these techniques, LDPI has drawn the most attention, and PSOCT is considered to have the most potential for the quantitative estimation of actual burn depth. ${ }^{2}$

LDPI estimates burn depth through a two-dimensional mapping of blood perfusion since damaged dermal vasculature results in a reduced blood flow. However, because it uses a laser with a long coherence length, LDPI cannot provide depth resolved information, and the correlation between the perfusion value and the burn depth is not straightforward. ${ }^{2}$ Moreover, LDPI is seriously affected by the optical heterogeneity of tissue.

PSOCT measures the depth directly by acquiring cross-sectional images of thermal injuries with high spatial resolution. It images the optical polarization changes in tissue that result from the thermal destruction of collagen fibers. But because PSOCT detects only the singly back-scattered photons, its maximum imaging depth in biological tissues is fundamentally limited to less than one optical transport mean free path $(\sim 1 \mathrm{~mm})$ due to strong optical scattering. This depth limit makes PSOCT unsuitable for imaging partial-thickness burns that are deeper than $1 \mathrm{~mm}$.

Consequently, a new noninvasive technique that provides high-resolution depth-resolved imaging of burns at a depth of a few millimeters offers great promise. Photoacoustic (PA) imaging is a hybrid volumetric imaging technology that images internal optical energy depositions by detecting laser-induced photoacoustic waves (PA waves) with an

\footnotetext{
*lhwang@biomed.wustl.edu; phone 1314 935-6152; fax 1314 935-7448; http://oilab.seas.wustl.edu
} 
ultrasonic detector. ${ }^{7}$ It combines high spatial resolution and large imaging depth with strong optical absorption contrast. Hence, PA imaging is potentially applicable to burn imaging so as to provide improved accuracy in measuring burn depth within the full depth range of partial-thickness burns. The feasibility of using PA profiling to evaluate burn depth has been demonstrated in rats. However, only poor spatial resolution has been achieved. ${ }^{8,9}$ Here, we apply photoacoustic microscopy $(\mathrm{PAM})^{10,11}$ to image acute thermal burns in pig skin.

\section{METHOD AND MATERIALS}

In biological tissues, oxyhemoglobin and deoxyhemoglobin are the dominating optical absorbers within a wide spectral range. Therefore, PAM is highly sensitive to blood. PAM measures burn depth based on imaging the hyperemia of acute thermal injuries, which have increased optical absorption than healthy and thermally coagulated tissues due to the higher blood content. ${ }^{12,13}$

The PAM system (figure1) used a tunable dye laser (ND6000, Continuum, CA) pumped by an Nd:YAG laser (Brilliant B, Bigsky, MT; pulse duration: $6.5 \mathrm{~ns}$; repetition rate: $10 \mathrm{~Hz}$ ) as the radiation source. The laser light at the 584-nm wavelength was delivered through an optical fiber. At the 584-nm optical wavelength, oxyhemoglobin and deoxyhemoglobin have identical molar extinction coefficients. Therefore, the optical absorption is sensitive only to the total hemoglobin concentration rather than to the different forms of hemoglobin oxygenation. This optical wavelength agreed with the previously demonstrated optimal spectral range in PA burn profiling. ${ }^{9}$ The light coming out of the fiber was first expanded by a conical lens and then weakly focused into samples by an optical condenser. An annular optical illumination was, therefore, formed on the sample surface with a dark center. The incident energy density on the sample surface, as well as around the optical focus, was controlled well within ANSI safety limits. ${ }^{14}$ A high-frequency ultrasonic transducer (V214-BB-RM, Panametrics, WA) with a custom-made spherical focusing lens was placed coaxially with the optical lenses. The focal region of the transducer and the optical focus overlapped each other to produce a confocal configuration. The PA signals received by the transducer were amplified by a wide-band low-noise amplifier (ZFL500LN, Mini-Circuits, NY) and were subsequently recorded by a digital oscilloscope (TDS 5034B, Tektronics, OR). In this study, 20 times signal averaging was applied to increase the signal-to-noise ratio for acquiring cross-sectional images.

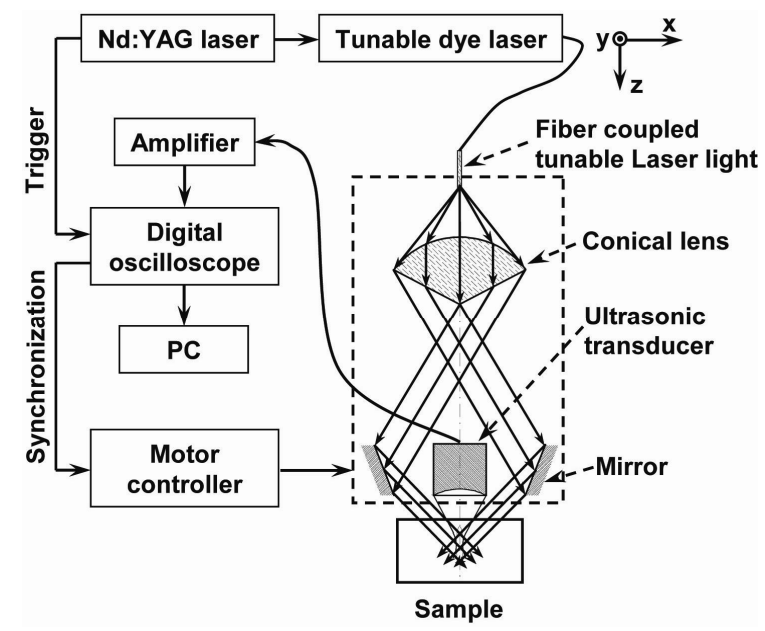

Fig. 1. Schematic of the PAM system.

To acquire an image, PAM recorded the PA wave for $2 \mu \mathrm{s}$ at each location of the ultrasonic transducer. After being normalized by the corresponding laser pulse energy, the time-resolved PA signal was converted into a one-dimensional depth-resolved image (A-line) based on the sound velocity in soft tissue $(1.54 \mathrm{~mm} / \mu \mathrm{s})$ along the $z$ axis. Next, rasterscanning in the horizontal $(x-y)$ plane with a step size of $50 \mu \mathrm{m}$ produced a three-dimensional image. Two types of twodimensional images were used in this work: (1) cross-sectional (B-scan) images along the $z-x$ plane; and (2) maximumamplitude-projection (MAP) images along the $x-y$ plane, where the maximum PA amplitude of each A-line was projected along the $z$ axis. 
The resolution of PAM is determined by the focal diameter and the bandwidth of the ultrasonic detector. Using an ultrasonic transducer with a center frequency of $50 \mathrm{MHz}$, a bandwidth of $80 \%$, and a numerical aperture of 0.44 , this PAM achieves an axial resolution of $15 \mu \mathrm{m}$ and a lateral resolution of $45 \mu \mathrm{m}$. Because a PA wave is generated by absorbed photons, PAM can image deeper than PSOCT by taking advantage of deeply-penetrating diffuse photons. At $50 \mathrm{MHz}$, the maximum imaging depth of PAM has been measured to be $3 \mathrm{~mm},{ }^{11}$ which is limited primarily by strong frequency dependent ultrasonic attenuation. The ratio of maximum imaging depth to axial resolution, which defines the number of effective pixels, of PAM is comparable to that of PSOCT and can achieve a value of more than 100. Moreover, PAM possesses an additional advantage in that its imaging depth can be increased by reducing the ultrasonic frequency. Although this results in degraded spatial resolution, the depth-to-resolution ratio can be preserved within certain ultrasonic frequency range.

We used White Yorkshire pigs (20 30 kg) with low skin melanin concentration as our animal model since pig skin has the closest anatomical resemblance to human skin. ${ }^{15}$ All experimental animal procedures were carried out in conformity with the guidelines of the $\mathrm{NIH} .{ }^{16}$ The laboratory animal protocol for this work was approved by the University Laboratory Animal Care Committee of Texas A\&M University.

After anesthetizing an animal with an intramuscular injection of xylazine at a dosage of $2.2 \mathrm{mg} / \mathrm{kg}$ and Telazol at a dosage of $5 \mathrm{mg} / \mathrm{kg}$, an area of $10 \times 10 \mathrm{~cm}^{2}$ on the pig back was depilated with commercial hair remover lotion. An acute burn was created by applying an electric cautery (Thermal Cautery Unit 150, Geiger Medical Technologies, IA; preheated to $175^{\circ} \mathrm{C}$ ) to the depilated skin area. After heating, edema and tissue coagulation were observed without obvious carbonization. A series of acute burns with different depths of damage were inflicted by varying the skin exposure to the cautery preheated to $150{ }^{\circ} \mathrm{C}$. During each heating period, the pressure applied to the skin through the cautery was kept constant. The burned skin together with a large area of adjacent normal skin and subcutaneous tissues were immediately excised to a depth of $10 \mathrm{~mm}$ for the PAM imaging. After the skin excision, the animals were euthanatized by an intravenous administration of pentobarbital at a dosage of $100 \mathrm{mg} / \mathrm{kg}$. All of the burns were classified by histological results as deep partial-thickness burns.

\section{RESULTS AND DISCUSSION}

Figure 2a shows a photograph of an acute skin burn that was heated at $175{ }^{\circ} \mathrm{C}$ for $20 \mathrm{~s}$ taken from the epidermal side after skin excision. A volume of $10 \times 10 \times 3 \mathrm{~mm}^{3}$ that covered the burn was imaged. Due to the optical and strong acoustic attenuations, the acquired PA signals from the hyperemic bowl on the skin surface had larger amplitudes than those from below. Therefore, the MAP image (figure $2 \mathrm{~b}$ ) shows only the hyperemic ring on the skin surface. For B-scan images, amplitude compensation along the $z$ axis was applied to better visualize the deeper structures. Since acoustic attenuation is the dominating factor at the 50-MHz center frequency, the measured PA amplitudes at difference depths beneath the skin surface were only compensated for based on the acoustic attenuation in porcine skin $\left(2.5 \mathrm{~dB} / \mathrm{cm} \mathrm{MHz}^{17}\right)$. This compensation provided a good first-order estimation of the true PA amplitudes generated from deep structures for morphological imaging purposes. The compensated B-scan image of the burn at the marked location in figure $2 \mathrm{~b}$ is given in figure $2 c$.

In a typical acute thermal injury, three distinct regions can be found: (1) a central zone of edema and coagulated tissue, where no blood flow is expected; (2) a red band called the hyperemic bowl (hyperemic ring when on the skin surface,) which results from hemorrhaging at the periphery of the coagulated zone; and (3) an outside thermally intact healthy tissue area. ${ }^{18}$ The hyperemic bowl represents the area in which hemostasis, hemorrhage, and hyperemia occur as a result of thermal damage to the blood vessels and the pathophysiologic response to heating. Hence it reflects the boundary of the thermal damage. These three zones are clearly observed in Fig. 2a. Because PAM is an optical absorption-based imaging modality and hemoglobin is the dominant optical absorbing pigment at the 584-nm wavelength, the contrast in the PAM imaging of acute burns comes from the differences in total hemoglobin concentration. Although increases in optical scattering in coagulated tissues over that in healthy tissues have been found, the optical absorption remains relatively constant around the 584-nm wavelength. ${ }^{12}$ For edema, both optical scattering and absorption decrease compared to normal tissue. ${ }^{19}$ As a result, the hyperemic bowl generates stronger PA waves due to higher hemoglobin concentration than do the healthy and thermally coagulated tissues. In Fig. 2b, the ratio of the imaged PA amplitude of the hyperemic ring to that of the inner coagulated tissue was 20.4 \pm 3.4 ; and the ratio of the imaged PA amplitude of the hyperemic ring to that of the outer normal tissue was 9.5 \pm 1.9 . Comparison with the photograph (figure $2 \mathrm{c}$ ) shows that both the morphology and the size of the hyperemic ring were correctly imaged by PAM. 


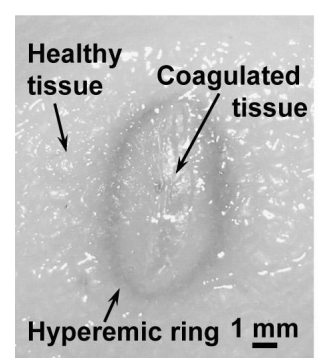

(a)

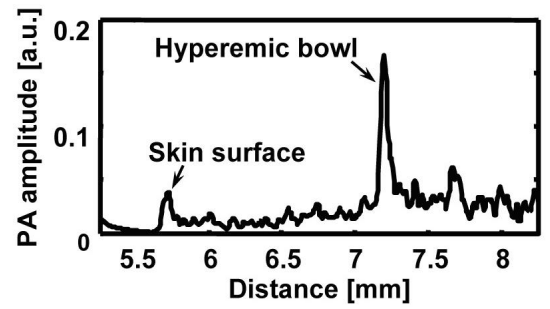

(e)

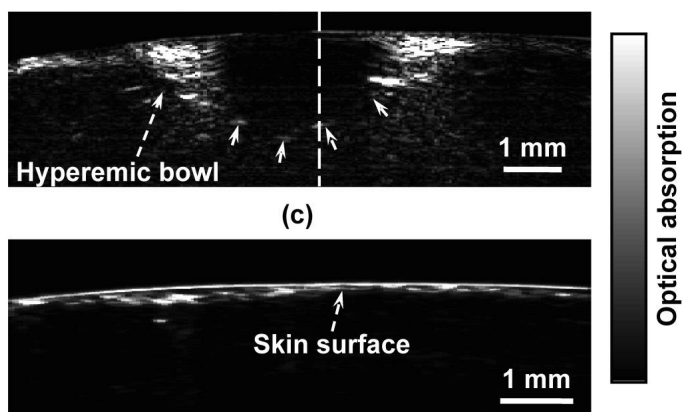

(d)

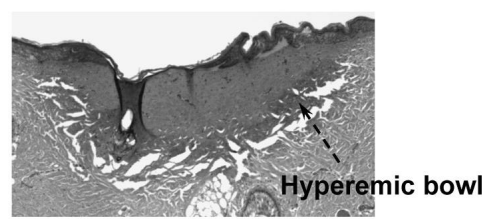

(f)

Fig. 2. PAM image of an acute skin burn heated at $175^{\circ} \mathrm{C}$ for $20 \mathrm{~s}$. (a) A photograph of the burn from the epidermal side. (b) A MAP image showing the morphology of the hyperemic ring. (c) The B-scan image of the hyperemic bowl at the location marked in panel B. (d) A typical B-scan image of healthy skin. (e) The A-line at the location marked in panel C. (f) A haematoxylin-and-eosin (HE) stained histological section.

In figure 2c, the middle dark region represents the thermally coagulated tissue, whose boundary is defined by the brighter hyperemic bowl as marked by the arrows. Because capillaries are beyond the spatial resolution of the current PAM system, no obvious structures were imaged in the outside healthy tissue in this burn sample. A typical B-scan of normal healthy skin is shown in figure $2 \mathrm{~d}$ for comparison after the same compensation for acoustic attenuation.

Once the B-scan image of the hyperemic bowl was acquired, the burn depth was estimated based on the distance between the imaged skin surface and the inner boundary of the hyperemic bowl. Figure $2 \mathrm{e}$ gives the A-line at the location marked in figure $2 \mathrm{c}$, where the horizontal axis represents the distance from the ultrasonic transducer. The two PA peaks in the A-line correspond to the skin surface and the inner boundary of the hyperemic bowl, respectively. Consequently, the distance between these two peaks was taken as an estimate of the burn depth. After a lateral neighborhood averaging within a region of $0.5 \mathrm{~mm}$ along the $x$ axis in figure 2c, the maximum burn depth was measured to be $1.73 \pm 0.07 \mathrm{~mm}$. A histological result showing the cross section of a hyperemic bowl is shown in figure $2 \mathrm{f}$.

Figures 3 a-d show the B-scan images of the acute skin burns that were heated at $150^{\circ} \mathrm{C}$ for $5,10,20$, and $30 \mathrm{~s}$, respectively. Based on the imaged inner boundaries of the hyperemic bowls, increased burn depth was observed for the longer thermal exposures, which agrees with the pathogenesis of acute burns. ${ }^{13}$ Moreover, the imaged width of the hyperemic bowls on the skin surface also increased with longer heating time, which was supported by direct observation before the skin excisions.

Compared to LDPI and PSOCT, which must correlate the measured parameters, such as blood perfusion and polarization changes, to the actual burn depth, PAM provides a direct assessment of the true boundaries of the burns by imaging the hyperemic bowl. Because vascular damage is a much more sensitive indicator than parenchymal change, e.g. denatured collagen, ${ }^{3}$ PAM has the potential to achieve high accuracy in assessing burn depth. Moreover, PAM is not affected by edema because edema introduces only small optical absorption changes relative to the strong optical absorption of hemoglobin. Some exceptions may exist in post acute burns and slow burns, where the boundaries of the thermal damage may not be well defined by the hyperemic bowl. ${ }^{5}$ Since the imaged burn depth measures up to $1.73 \pm 0.07 \mathrm{~mm}$, PAM is well suited for imaging human skin. 


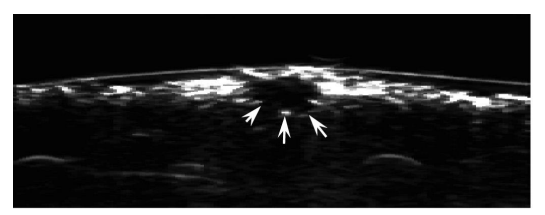

(a)

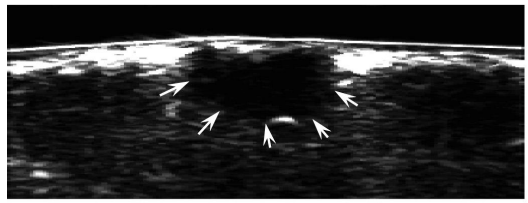

(c)

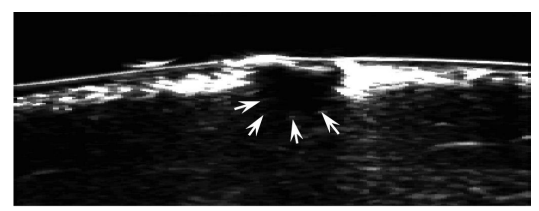

(b)

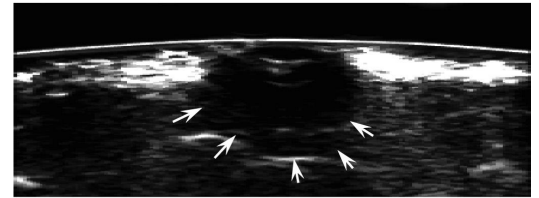

(d)

Fig. 3. PAM images of acute skin burns with different depths introduced by changing the heating durations to 5 (a), 10 (b), 20 (c), and 30 (d) seconds.

In this study, due to the lack of flexibility of the desk-top PAM system, the acute burns were imaged immediately after skin excision, and a single optical wavelength at the isosbestic point was used to demonstrate the feasibility. However, PAM itself is a noninvasive technology and a hand-held version of PAM has been developed, which is operated similarly to a traditional ultrasound imager to enable us to perform in vivo studies. To better understand the pathophysiology of burn injuries, hemoglobin oxygen saturation will be measured using multi-wavelength PAM imaging in vivo. ${ }^{11}$ A time course study of the change in hemoglobin oxygen saturation will also be conducted to investigate post burn reactions and to examine healing. ${ }^{20}$

There are two technical limitations to the current PAM. First, because of the low laser repetition rate $(10 \mathrm{~Hz})$, data acquisition for a B-scan image (20 times averaging) takes 10 minutes. A new laser system with a pulse repetition rate of up to $1 \mathrm{kHz}$ has been acquired, which enables a frame rate of $1 \mathrm{~Hz}$ using our new handheld PAM system. The second limitation is that PAM requires a coupling medium (Ultrasound scanning gel, Sonotech, WA) between the ultrasonic detector and the damaged tissues in order to detect the induced PA waves. This contact may possibly create difficulties in clinical environments. However, no mechanical pressure was applied through the ultrasound gel, and commercially available sterilized ultrasound gel could be used to prevent infections.

\section{SUMMARY}

In summary, the feasibility of imaging acute burns and thus determining their depths using PAM has been demonstrated by imaging the hyperemic bowl. High imaging contrast and resolution has been achieved in three dimensions based on the differences in total hemoglobin concentration and strong ultrasonic focusing. Burn depths of up to $1.73 \pm 0.07 \mathrm{~mm}$ have been imaged, and increased burn depth related to longer heating period has been observed. In addition to structural imaging, functional imaging is possible by performing spectral measurements in vivo.

We thank Dr. Geng Ku and Xueyi Xie for experimental assistance. This project is sponsored by National Institutes of Health grants R01 EB000712 and R01 NS46214.

\section{REFERENCES}

1. D. Heimbach, L. Engrav, B. Grube, and J. Marvin, "Burn depth: a review,” World J. Surg. 16, 10-15 (1992).

2. B. S. Atiyeh, S. W. Gunn, and S. N. Hayek, "State of the art in burn treatment," World J. Surg. 29, 131-148 (2005).

3. A. Watts, M. Tyler, M. Perry, A. Roberts, and D. McGrouther, "Burn depth and its histological measurement," Burns 27, 154$160(2001)$.

4 D. Park, J. Hwang, K. Jang, D. Han, K. Ahn, and B. Baik, "Use of laser Doppler flowmetry for estimation of the depth of burns," Plast. Reconstr. Surg. 101, 1516-1523 (1998).

5 S. Srinivas, J. de Boer, H. Park, K. Keikhanzadeh, H. Huang, J. Zhang, W. Jung, Z. Chen, and J. Nelson, "Determination of burn depth by polarization-sensitive optical coherence tomography," J. Biomed. Opt. 9, 207-212 (2004). 
6. S. Jiao, W. Yu, G. Stoica, and L. V. Wang, "Phase-based polarization contrast Mueller optical coherence tomography and application in burn imaging," Appl. Opt. 42, 5191-5197 (2003).

7. X. Wang, Y. Pang, G. Ku, X. Xie, G. Stoica, and L. V. Wang, "Non-invasive laser-induced photoacoustic tomography for structural and functional imaging of the brain in vivo ," Nature Biotech. 21, 803-806 (2003).

8. S. Sato, M. Yamazaki, D. Saitoh, H. Tsuda, Y. Okada, M. Obara, and H. Ashida, "Photoacoustic diagnosis of burns in rats," J. Trauma 59, 1450-1455 (2005).

9. M. Yamazaki, S. Sato, H. Ashida, D. Saito, Y. Okada, and M. Obara, "Measurement of burn depth in rats using multiwavelength photoacoustic depth profiling," J. Biomed. Opt. 10, 064011 1-4 (2005).

10. K. Maslov, G. Stoica, and L. V. Wang, "In vivo dark-field reflection-mode photoacoustic microscopy," Opt. Lett. 30, 625-627 (2005).

11. H. F. Zhang, K. Maslov, G. Stoica, and L. V. Wang, "Functional photoacoustic microscopy," Nature Biotech. 24, 848-851 (2006)

12. S. Thomsen, H. Vijverberg, R. Huang, and J. Schwartz, "Changes in optical properties of rat skin during thermal coagulation," Proceedings of the SPIE 1882, 230-236 (1993).

13. A. R. Moritz, "Studies of thermal injury III. The pathology and pathogenesis of cutaneous burns an experimental study," Am. $J$. Pathol. 23, 915-941 (1947).

14. American National Standards Institute, American national standard for the safe use of lasers, ANSI Standard Z136.1-2000, ANSI, Inc., New York, NY, 2000.

15. A. R. Moritz, and F. C. Henriques, "Studies of thermal injury II. The relative importance of time and surface temperature in the causation of cutaneous burns," Am. J. Pathol. 23, 695-720 (1947).

16. National Institutes of Health, Guide for the Care and Use of Laboratory Animals, NIH Pub. 86-23, U.S. Government Printing Office, Washington DC, 1985.

17. T. Baldeweck, P. Laugier, and G. Berger, "An in vitro study on porcine skin: attenuation profile estimation using auto-regressive modeling," IEEE Proceedings of Ultrasonic Symposium 1995 2, 1141-1144 (1995).

18. B.-M. Kim, S. L. Jacques, S. Rastegar, S. Thomsen, and M. Motamedi, "Nonlinear finite-element analysis pf the role of dynamic changes in blood perfusion and optical properties in laser coagulation of tissue," IEEE J. Sel. Top. Quant. Electron. 2, 922-933 (1996).

19. Y. Ueda, S. Sato, H. Ashida, H. Ooigawa, H. Nawashiro, K. Shima, D. Saitoh, Y. Okada, and M. Obara, "Transcranial measurement of diffuse light reflectance from cold-injured brains in rats," J. Biomed. Opt. 10, 064010-1-7 (2005).

20. A. Tandara, and T. Mustoe, "Oxygen in wound healing—-more than a nutrient," World J. Surg. 28, 294-300 (2004). 